\title{
Functional analysis of the Landsberg erecta allele of FRIGIDA
}

\author{
Inga Schmalenbach ${ }^{1 \dagger}$, Lei Zhang ${ }^{1 \dagger}$, Malgorzata Ryngajllo ${ }^{1}$ and José M Jiménez-Gómez ${ }^{1,2^{*}}$
}

\begin{abstract}
Background: Most of the natural variation in flowering time in Arabidopsis thaliana can be attributed to allelic variation at the gene FRIGIDA (FRI, AT4G00650), which activates expression of the floral repressor FLOWERING LOCUS C (FLC, AT5G10140). Usually, late-flowering accessions carry functional FRI alleles (FRI-wt), whereas early flowering accessions contain non-functional alleles. The two most frequent alleles found in early flowering accessions are the ones present in the commonly used lab strains Columbia (FRI-COI) and Landsberg erecta (FRI-Ler), which contain a premature stop codon and a deletion of the start codon respectively.

Results: Analysis of flowering time data from various Arabidopsis natural accessions indicated that the FRI-Ler allele retains some functionality. We generated transgenic lines carrying the FRI-Col or FRI-Ler allele in order to compare their effect on flowering time, vernalization response and FLC expression in the same genetic background. We characterize their modes of regulation through allele-specific expression and their relevance in nature through re-analysis of published datasets. We demonstrate that the FRI-Ler allele induces FLC expression, delays flowering time and confers sensitivity to vernalization in contrast to the true null FRI-Col allele. Nevertheless, the FRI-Ler allele revealed a weaker effect when compared to the fully functional FRI-wt allele, mainly due to reduced expression.

Conclusions: The present study defines for the first time the existence of a new class of Arabidopsis accessions with an intermediate phenotype between slow and rapid cycling types. Although using available data from a common garden experiment we cannot observe fitness differences between accessions carrying the FRI-Ler or the FRI-Col allele, the phenotypic changes observed in the lab suggest that variation in these alleles could play a role in adaptation to specific natural environments.
\end{abstract}

Keywords: Arabidopsis thaliana, Flowering time, FRIGIDA, Vernalization, Natural variation, Allelic series

\section{Background}

As plants are sessile organisms, adaptation to the environment is essential for their survival and reproductive success. Mechanisms regulating the response to environmental cues enable a proper timing of key events in a plant's life. One crucial event, resulting from the integration of endogenous and environmental signals, is the switch from vegetative to reproductive development. The annual species Arabidopsis thaliana occurs in the northern hemisphere in a broad range of latitudes differing substantially in day length, temperature and other ecological factors [1]. As a result of adaptation to specific habitats, Arabidopsis accessions have

\footnotetext{
*Correspondence: jmijmenez@mpipz.mpg.de

${ }^{\dagger}$ Equal contributors

'Department of Plant Breeding and Genetics, Max Planck Institute for Plant Breeding Research, Carl-von-Linné-Weg 10, 50829 Cologne, Germany

${ }^{2}$ INRA, UMR1318, Institut Jean-Pierre Bourgin, RD10, F-78000 Versailles, France
}

evolved two main life history strategies. Winter-annuals germinate in autumn, survive winter as a rosette and flower in the following summer, whereas summer-annuals germinate in spring or summer and finish their reproduction cycle in the same year. Variation between these distinct strategies has been associated with allelic variation at the genes FRIGIDA (FRI) and FLOWERING LOCUS C (FLC), which act epistatically to regulate flowering time [2,3]. Arabidopsis individuals containing functional alleles at these two loci flower very late or not at all, unless they receive a prolonged exposure to cold (vernalization).

FLC encodes a MADS-box transcription factor that binds to the promoters of floral initiators such as FLOWERING LOCUS T (FT) and SUPPRESSOR OF CONSTANS OVEREXPRESSION 1 (SOC1) to repress their transcription. Flowering occurs when $F L C$ is downregulated by proteins of the vernalization and/or autonomous pathways, 
reviewed in [4-7]. Transcriptional activation of FLC requires integrated activity of diverse chromatin remodeling and histone-modifying complexes, reviewed in [8]. When Arabidopsis plants are vernalized, expression of $F L C$ is decreased and maintained at reduced level by different epigenetic marks in a Polycomb-mediated process involving long non-coding RNAs [9-11]. The epigenetic silencing of $F L C$ is quantitatively modulated and underlies Arabidopsis natural variation for vernalization response [12-14]. Two main haplogroups of FLC have been defined mainly by polymorphisms within the first intron, an important region to maintain silencing induced by vernalization $[15,16]$. These haplogroups were shown to underlie differences in flowering time among natural accessions of Arabidopsis, but only when a functional FRI allele is present [15].

Despite the central role of $F L C$, most of the variation in flowering time has been found to correlate with natural allelic diversity at FRI [3]. FRI is the founding member of a family of seven Arabidopsis proteins that contain two coiled-coil domains and show no homology to other proteins [2,17]. In order to regulate flowering time, $F R I$ builds the scaffold protein of a transcription activator complex that mediates diverse chromatin modifications at $F L C[18,19]$. Furthermore, FRI is suggested to be involved in co-transcriptional processes that link the function of $5^{\prime}$ end capping with transcription and efficient splicing of FLC [20]. Vernalization abolishes the effect of FRI and silences $F L C$ as described above $[9,12]$.

A considerable number of different FRI haplotypes have been identified within accessions from a wide range of latitudes $[14,21,22]$ or more restricted geographic regions $[23,24]$. Studies on these alleles led to the conclusion that early flowering types evolved by multiple independent mutational events from winter-annuals containing an ancestral functional $F R I$ allele (FRI-wt) [25]. Two distinct deletions in FRI are believed to confer early flowering in most of the rapid cycling accessions. The Columbia allele (FRI-Col) carries a 16 bp-deletion resulting in a premature stop codon and, thus, a truncated protein missing a part of the C-terminal $[2,14]$. But the most frequent deleterious FRI mutation in nature is a $376 \mathrm{bp}$-deletion combined with a $31 \mathrm{bp}$-insertion in the promoter as observed in Landsberg erecta (FRI-Ler) $[14,21,22]$. This mutation disrupts the translational start but, due to a second alternative start codon, a short out-of-frame protein might be built [2]. The loss-offunction FRI alleles found in Ler and Col are widely used as examples of positive selection towards rapid cycling accessions [26,27].

Although the FRI-Ler and FRI-Col alleles are always classified as a single non-functional group, there are evidences for differences in their effects. First, accessions carrying the Ler-type deletion but not the Col-type deletion show considerable variation in flowering time and $F L C$ levels $[14,28]$. Then, several studies find variation in flowering time associated with the chromosomal region of $F R I$ in mapping populations derived from accessions containing the FRI-Ler allele crossed to accessions with a loss-of-function allele [28-30]. In all these cases, the Ler allele was associated with a delay in flowering time. This delay was attributed to additional loci in the same region as FRI, although these have never been identified.

This article provides the first robust evidence that the Ler allele of $F R I$ is functional. In contrast to the true null FRI-Col allele, the FRI-Ler allele is able to induce FLC expression, resulting in delayed flowering and increased vernalization sensitivity. Nevertheless, the FRI-Ler allele has a weaker effect than the fully functional FRI-wt allele. The reduced functionality of the FRI-Ler allele mainly results from its lower expression putatively due to cis regulatory polymorphisms in its promoter. Our finding defines a new functional class of FRI alleles, in which accessions carrying the FRI-Ler allele would flower in between the late and early flowering groups defined so far.

\section{Results}

Effects of the FRI-Ler and FRI-Col alleles on flowering time and vernalization response

We investigated if the FRI alleles present in Col-0 and Ler show evidences of different functionality by analyzing flowering time data from a recombinant inbred line (RIL) population derived from a cross between the two accessions [31]. Because the effect of FRI on flowering time depends on the alleles at $F L C$, we took into account the genotype of the RILs at both loci [15]. While Col-0 harbors a functional FLC allele, Ler has been described to contain a weak allele with a transposon-like insertion in the first intron $[25,32,33]$. As shown in Figure 1a, plants carrying the FRI-Ler allele flower significantly later than plants carrying the FRI-Col allele, although only in the presence of the strong FLC allele of Col-0 (two-way ANOVA, FRI $\mathrm{p}=0.0095, F L C \mathrm{p}=0.0138$, interaction $\mathrm{p}=0.0824)$. This epistatic interaction suggests the existence of a functional FRI allele in Ler, as no other gene located in this region of chromosome 4 has been shown to delay flowering time through interactions with a locus in the region of chromosome 5 containing FLC.

Because strong vernalization requirements are associated with functional $F R I$ alleles, we decided to analyze variation in vernalization sensitivity among Arabidopsis accessions $[34,35]$. We calculated the decrease in flowering time in response to vernalization in a published dataset containing 126 Arabidopsis accessions classified as FRI-Col, FRI-Ler or FRI-wt [22]. Accessions carrying the FRI-Ler allele accelerated flowering by 10 days in response 


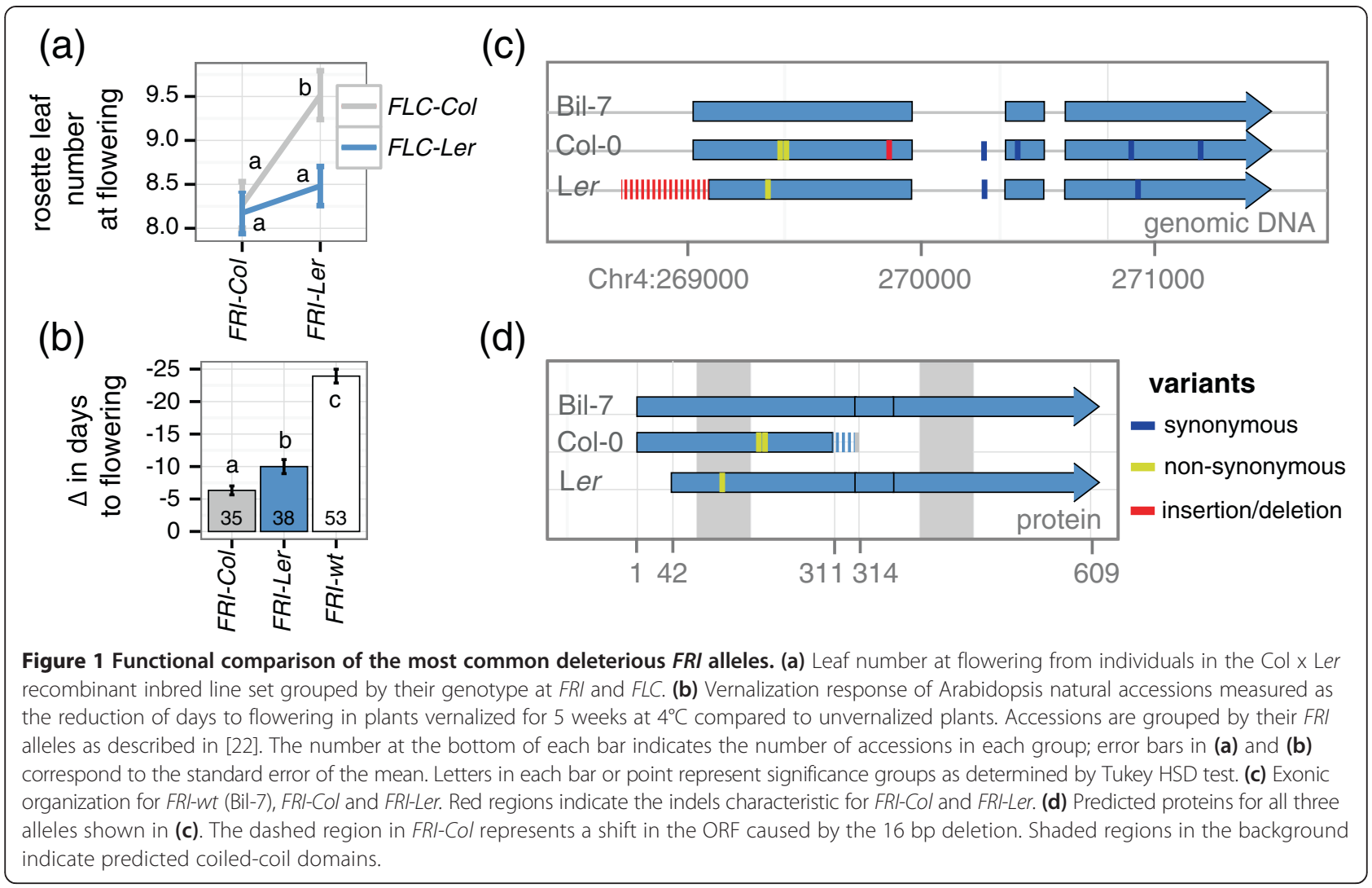

to vernalization (Figure 1b). This acceleration was intermediate to the low response of accessions containing the FRI-Col deletion and the high response of accessions carrying the FRI-wt allele (Figure $1 \mathrm{~b}$ ). The same trend was found for the vernalization response quantified as total leaf number, although the difference between accessions carrying the FRI-Ler allele and the FRI-Col allele was not statistically significant (Additional file 1).

Taken together, our results disagree with the common belief that the FRI allele present in Ler is not functional. However, from this analysis we cannot completely rule out the possibility that the observed phenotypes are caused by additional loci in linkage disequilibrium with FRI.

\section{Structural characterization of the FRI-Ler and FRI-Col alleles}

We looked for further evidences of a functional FRI-Ler allele by studying sequence variation among Arabidopsis accessions. We sequenced genomic DNA from FRI including its upstream and downstream regions in Col-0, Ler-1 and Bil-7, the latter being a winter-annual accession that contains a functional FRI-wt allele identical to the published H51 allele [2,14]. All polymorphisms found in FRI-Col and FRI-Ler correspond exactly to the ones described before, such as the insertion/deletions that define these two allelic classes (Figure 1c; $[2,14])$. The H51 allele is predicted to encode a fully functional 609 aa protein [GenBank:
AAG23415] while the Col allele yields a 314 aa protein [GenBank: AEE81913], truncated due to a premature stop codon resulting from a 16 bp deletion in exon 1 (Figure 1c and d; [2]). In the case of FRI-Ler, a 376 bp deletion combined with a 31 bp insertion removes the translational start, but creates a new, out-of-frame start codon that is predicted to yield a 41 aa protein [2]. Interestingly, in addition to the out-of-frame start codon, FRI-Ler contains an inframe ATG codon downstream of the original start, which would result in a protein missing 42 aa of the N-terminus. Apart from this deletion and one conservative amino acid change from $\mathrm{L}$ to I in the first coiled-coil domain, the FRILer protein is identical to the functional Bil-7 allele (Figure 1d). There are no known functional motifs in the deleted segment of the FRI protein, and, thus, if transcription and translation occurred from the downstream inframe start codon, the resulting FRI-Ler protein could possibly be as functional as the full-length Bil-7 protein.

We looked for evidences of transcription of this long FRI-Ler allele using RNA-seq reads from seven accessions containing the characteristic Ler indel [36]. We found signals of expression across the full length of the gene, including the C-terminal part, in all accessions (see Additional file 2). This raised the question of whether this long FRI-Ler transcript is translated into a protein and if so, whether it is sufficient to delay flowering and to confer vernalization sensitivity. 


\section{Comparing the effect of the FRI-Ler versus the FRI-Col allele in transgenics}

We studied the existence of a functional FRI-Ler allele by comparing its function with that of the FRI-Col allele in transgenic plants. For this, we cloned the putative coding region plus the upstream and downstream region of both alleles and transformed them in a common background. We chose Col-0 as a recipient because our previous results suggest that FRI-Col carries a loss-of-function mutation (Figure 1), and because Col-0 has been shown to contain a strong FLC allele that is activated in the presence of a FRI$w t$ allele [3]. First, we confirmed the lack of function of the FRI-Col allele by growing three independent $\mathrm{T}_{3}$ lines homozygous for the FRI-Col transgene (Col-0[FRI-Col $]$ ) under long day conditions in the greenhouse. In this experiment none of the transgenic lines was significantly different from wild type (Figure 2a). In a consecutive experiment in similar conditions we grew homozygous $\mathrm{T}_{3}$ transgenic lines containing the FRI-Ler transgene (Col-0[FRI-Ler]) and observed a significant delay in flowering in all lines compared with the Col-0 wild type (Figure 2b), confirming FRI-Ler functionality.

Because FRI delays flowering time through upregulation of the floral repressor FLC [3], we performed qRT-PCR to test the expression of FLC in the transgenic plants. As expected, we found elevated expression of FLC associated with the FRI-Ler transgene but not with the FRI-Col transgene (Figure 2c), despite all lines presenting increased FRI expression (Additional file 3). In addition, a prolonged cold treatment, known to abolish FLC expression, significantly reduced leaf number in Col-0[FRI-Ler] lines, but not in Col-0 wild type or in Col-0[FRI-Col] lines ( $\mathrm{p}<0.001, \mathrm{p}=0.06$ and $\mathrm{p}=0.28$ for the interaction between vernalization and genotype in a two-way ANOVA respectively, compare Figure $2 \mathrm{~d}$ and Figure $2 \mathrm{a}$ and $\mathrm{b}$ ).

Altogether, our results demonstrate that the FRI-Ler allele, but not the FRI-Col allele, has the ability to delay flowering, and that this delay is achieved through upregulation of FLC.

\section{Expression of $F R I$ and $F L C$ in natural accessions}

The functionality of the FRI-Ler allele is contrasting with its frequent presence among early flowering accessions $[2,14]$. Our analyses also show that, although accessions carrying FRI-Ler alleles have stronger vernalization responses than accessions containing FRI-Col alleles, they do not reach the levels observed in lines carrying FRI-wt alleles (Figure $1 \mathrm{~b}$ and Additional file 1). This suggests a reduced functionality of the FRI-Ler allele in comparison with the FRI-wt allele, which can be caused by differences in the protein sequence, in expression levels or in post-transcriptional/post-translational mechanisms. Previous works have pointed to the low expression of the FRI-Ler allele, possibly as a consequence of the indel in
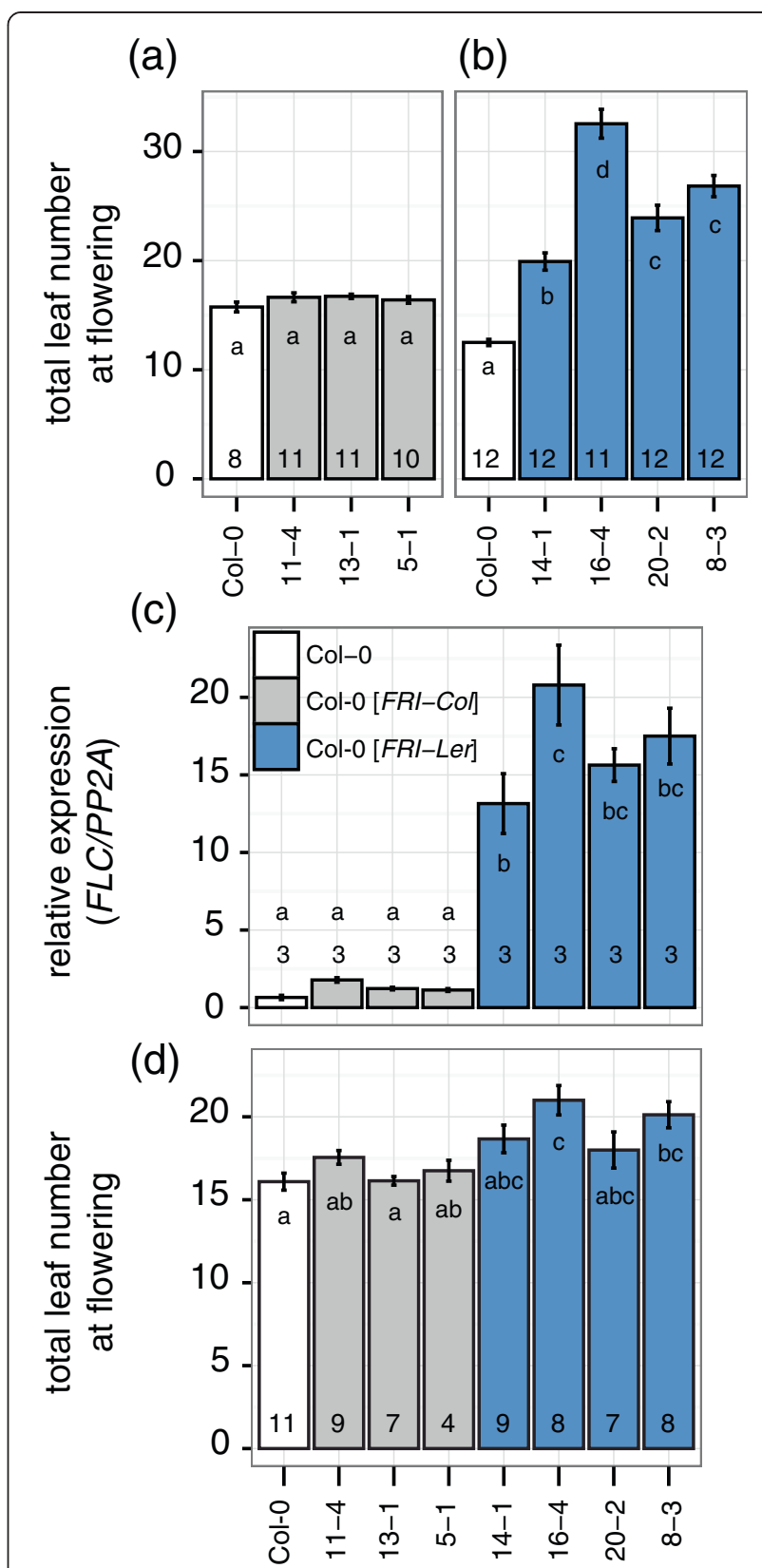

Figure 2 Characterization of transgenic lines carrying the FRI-Col or FRI-Ler allele. (a) Flowering time expressed as total leaf number (rosette + cauline) from homozygous $T_{3}$ single insertion lines containing a FRl-Col transgene are shown in comparison to the untransformed Col0 wild type. (b) Experiment as in (a) but using homozygous $T_{3}$ single insertion lines transformed with FRI-Ler. (c) Expression of FLC for the same genotypes as above. In this experiment, leaf tissue was collected from 10 day-old seedlings grown in long day conditions. The number in each bar indicates the number of biological replicates used. Expression was normalized to the expression of PP2A. (d) Flowering time after vernalization quantified as total leaf number for the same lines as above. Plants were vernalized for four weeks at $4^{\circ} \mathrm{C}$ and subsequently grown under long day conditions. The number in each bar indicates the number of individual plants per line analyzed. Error bars represent the standard error of the mean. Letters in each bar represent significance groups as determined by Tukey HSD test. 
its promoter [2,37]. This led us to focus on a possible transcription inhibition as the cause for early flowering in natural accessions containing this allele.

We analyzed the expression profiles of $F R I$ and its downstream target FLC in 137 Arabidopsis accessions containing FRI-Ler, FRI-Col or FRI-wt alleles [38]. Accessions carrying FRI-Ler alleles showed significantly reduced expression of $F R I$ when compared to accessions carrying FRI-Col or FRI-wt alleles, suggesting a regulatory defect in the FRI-Ler allele (Figure 3a). Interestingly, this residual FRI-Ler expression results in higher FLC levels than in the accessions carrying FRI-Col alleles, although this difference is not significant (Tukey HSD test $\mathrm{p}$ value $\mathrm{p}=0.079$ ), possibly due to the large variation in $F L C$ expression found among accessions with FRI-Ler alleles (compare error bars in Figure 3b).

\section{Allele-specific expression of FRI-Ler and FRI-Col}

There are two alternative causes for the lower FRI expression found in Arabidopsis accessions carrying the FRI-Ler allele when compared to accessions carrying FRI-wt or FRI$\mathrm{Col}$ alleles. Either the FRI-Ler allele contains a mutation in cis, such as the promoter indel that defines the class, or its reduced expression is caused by the effect of transregulators that may occur in the early flowering accessions. In order to distinguish among these possibilities, we analyzed the allele specific expression of FRI using pyrosequencing in hybrids from crosses of Arabidopsis accessions differentially expressing this gene. Because expression of both alleles in a hybrid cell is controlled by the same set of regulators, the relative abundance of each allele in the hybrid should be equaled out if trans-regulators cause the differences between accessions. In contrast, if the expression differences between accessions are caused by mutations in cis, the differences in the expression of the alleles in the hybrid will be maintained [39].

We generated $F_{1}$ hybrids by crossing Ler- 1 with Col- 0 and with four accessions containing FRI-wt alleles (Bor-4, Bur-0, Sha and Knox-10; [14]). In all $F_{1}$ individuals analyzed the relative abundance of each allele measured in genomic DNA was close to $50 \%$, which ensured that the assay had no preference for either of the two alleles (Figure 3c). Analysis of cDNA from the same individuals revealed lower relative abundance of FRI-Ler than either the FRI-wt or the FRI-Col alleles (Figure 3d). These differences suggest that at least part of the phenotypic differences found between accessions carrying the FRI-Ler allele and those carrying the FRI-wt allele is due to a cis regulatory mutation reducing the expression of FRI-Ler. Interestingly, the differences in expression found among accessions classified by their $F R I$ allele are larger than the differences observed in allele specific expression in the hybrids (compare Figure 3a and d), suggesting the existence of additional trans-regulators controlling differences in expression among alleles. Further experiments will be required to determine the precise mode of regulation of this gene.

\section{Effect of coding polymorphisms in FRI-Ler}

In addition to the suggested regulatory polymorphisms causing inhibition of the expression of the FRI-Ler allele, its predicted protein differs from the FRI-wt protein in the deletion of 42 amino acids from the $\mathrm{N}$ terminal and in one substitution located in a coiled-coil domain (Figure 1d). To test whether these coding differences have an effect on the function of the FRI-Ler protein compared to FRI-wt, we
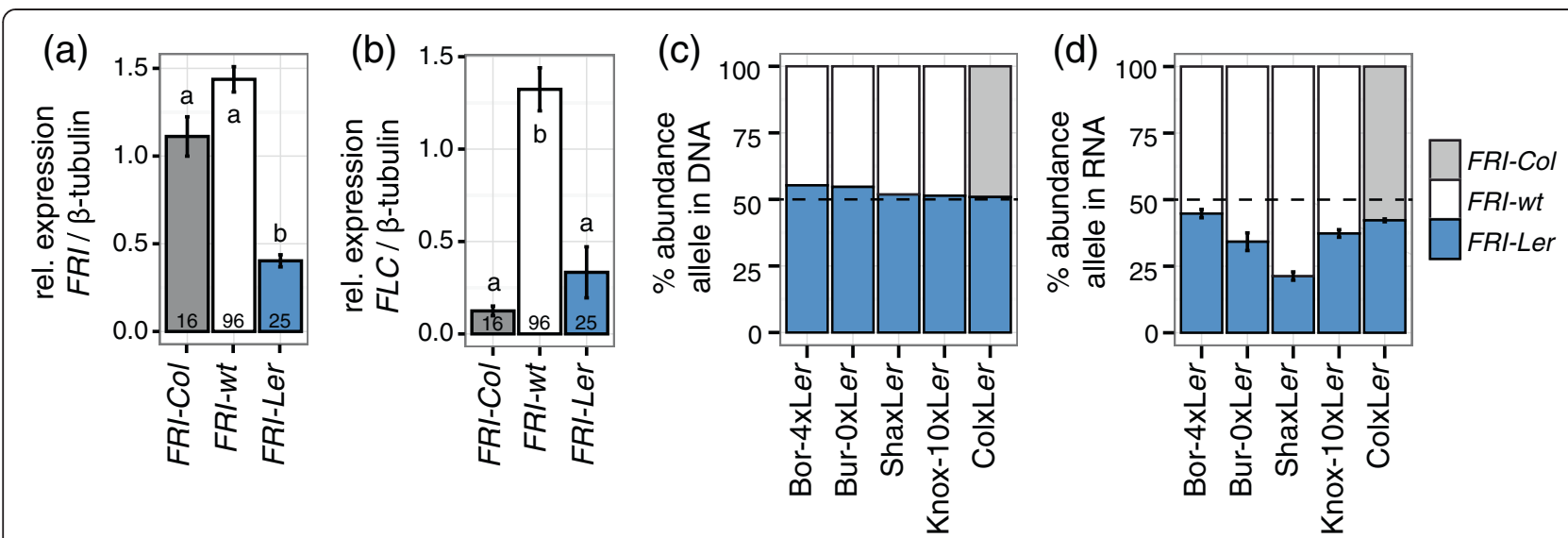

Figure 3 Relative expression levels of $F R I$ and $F L C$ in Arabidopsis accessions and $\mathrm{F}_{1}$ hybrids grouped by their $F R I$ allele. (a and b) 137 Arabidopsis accessions were grown in long days under greenhouse conditions, sampled after four weeks and expression levels of FLC and FRI were quantified from northern hybridizations [38]. Letters in each bar indicate the significance groups as determined by a Tukey HSD test. The number in each bar indicates the number of accessions in each group. (c and d) Positions with SNPs specific to Ler-1 or Col-0 were targeted using pyrosequencing in genomic DNA (c) or in CDNA (d) of $F_{1}$ hybrids. Leaf material for hybrids involving Bor-4, Bur-0, Knox-10 or Sha was collected at the time of bolting from plants growing under 12-hour photoperiods in an environmental chamber. Leaf material from hybrids involving Col-0 was collected from 10 day-old seedlings grown in the greenhouse in long days. Error bars indicate the standard error of the mean. 
generated transgenic lines carrying constructs in which the coding region of each allele was placed downstream of the native promoter of the other allele (Figure 4). Due to constraints (see Material and Methods), this experiment was performed using $\mathrm{T}_{1}$ lines carrying FRI-wt (the FRI-wt allele expressed under its native promoter), FRI-wt::FRI-Ler (the FRI-Ler allele expressed under the FRI-wt promoter) or FRI-Ler::FRI-wt (the FRI-wt allele expressed under the FRI-Ler promoter) constructs, and individuals from two independent $\mathrm{T}_{3}$ lines carrying the FRI-Ler construct (the FRI-Ler allele expressed under its native promoter). As expected, lines carrying the wild type promoter driving the expression of the wild type allele $(F R I-w t)$ flowered with the same number of leaves as Col FRI-Sf2, a line in which the FRI-wt allele from the accession $\mathrm{Sf}-2$ was introgressed into Col-0 [2]. In addition, lines carrying constructs with the FRI-Ler promoter flowered significantly earlier than those with alleles driven by the FRI-wt promoter. This supports our hypothesis that cis-regulatory elements specific to the FRI-Ler promoter are the cause of early flowering in accessions carrying this allele. Interestingly, lines carrying the FRI-Ler coding region flowered earlier than lines carrying the FRI-wt coding region when expressed under the same promoter. Although this acceleration of flowering was not significant, it was observed both when using the FRI-Ler promoter and the FRI-wt promoter. This result leaves open the possibility that the loss of 42 amino acids and/or the mutation in the coiled coil domain in the FRI-Ler protein contribute to accelerating flowering in the accessions that express it.

\section{Analysis of life history parameters in the FRI-Col and FRI-Ler accession groups}

A major question that derives from the classification of FRI-Ler as a semi-functional allele is whether it represents a change in the life history of the accessions that carry it when compared to the accessions carrying the FRI-Col allele. Although FRI-Ler accessions revealed a significant higher response to vernalization than accessions with the FRI-Col allele (Figure 1b), both groups only include earlyflowering accessions that are not likely to differ in their general life history strategy. Nevertheless, we investigated the variation in specific life history traits between the two allelic classes, which can result in a distinct ability to adapt to local environments. To address this question, we reanalyzed fitness-related phenotypes for a panel of accessions grown at four different field locations [40]. Although we observed small fitness differences in accessions carrying the FRI-Col and FRI-Ler alleles at various locations, these were not significant (see Additional file 4). On the other hand, accessions containing the FRI-wt allele were often significantly different in fitness traits when compared with FRI-Ler and FRI-Col. Further experiments will be required to determine if differences between the FRI-Ler

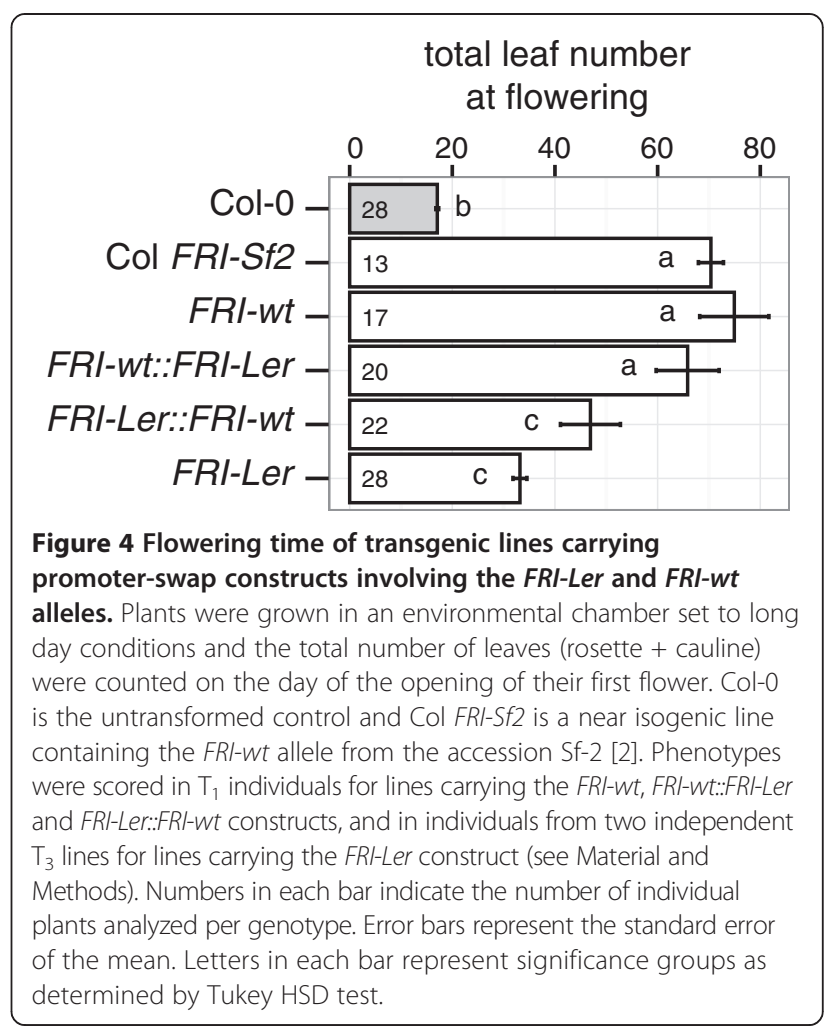

and $\mathrm{FRI-Col}$ allelic classes are relevant in specific environmental conditions (e.g. under stress).

\section{Discussion}

$F R I$, an activator of the negative regulator $F L C$, is the major determinant of natural variation in flowering time and vernalization sensitivity in Arabidopsis thaliana [3]. So far, natural allelic variants of $F R I$ have been clustered into two groups, the functional FRI-wt group and the non-functional group that included the FRI-Col and the FRI-Ler alleles. Here we prove that FRI-Ler, the most common allele found so far in summer-annual accessions, is partially functional, although it is a weak allele compared to the FRI-wt allele present in winter-annual types. We suggest that the indel found in FRI-Ler, including part of the promoter and the beginning of the coding sequence, allows transcription of an active messenger from a downstream methionine. This resulting transcript shows lower expression and perhaps slightly reduced activity than the transcripts from both FRI-wt and FRI-Col, but has the ability to up regulate FLC, delay flowering time and confer vernalization sensitivity. In contrast, the FRI-Col allele is unable to confer any of these phenotypes, despite being expressed at a level similar to the FRI-wt alleles present in winter-annual accessions.

Although FRI has a prominent role, not all variation in $F L C$ expression and flowering time observed in Arabidopsis accessions can be explained by variation at this gene. Previous studies have demonstrated that allelic 
variation at $F L C$ itself provides a basis for the development of a summer-annual flowering time habit $[24,25,41]$. As shown by [15], variation in flowering time may be associated with FLC only in the presence of a functional FRI allele. Consistent with this, we observed that accessions carrying the FRI-Ler or FRI-wt alleles, but not those carrying a FRI-Col allele, varied considerably in FLC expression and flowering time (Figures 1 and 3). Further variation of $F L C$ levels can be due to additional factors such as mutations in genes of the autonomous floral promotion pathway [25,42-44]. Moreover, allelic variation at genes directly interacting with $F R I$, namely SUPPRESSOR OF FRIGIDA 4 (SUF4), FRIGIDA-LIKE 1 (FRL1), FRIGIDA ESSENTIAL 1 (FES1) and FLC EXPRESSOR (FLX), could have an impact [18]. For instance, it has been shown that Ler contains a non-functional FRL1 allele, which is compensated by a functional FRIGIDA-LIKE 2 (FRL2) allele [45]. The latter restores FRImediated up regulation of $F L C$ expression and, thus, a late flowering phenotype. In contrast, Col- 0 carries a functional $F R L 1$ allele able to interact with $F R I$, but an impaired FRL2 allele [45].

The effect on flowering time, FLC expression and vernalization response conferred by the FRI-Ler allele observed in the present study is not comparable to the effect of the FRI-wt allele. Although we show here that both alleles differ greatly in expression (Figure 3 ), we detect small, non-significant but consistent differences between the FRI-Ler and the FRI-wt allele driven under the same promoter (Figure 4). This suggests that the putative truncation of the first 42 aa of the $\mathrm{N}$-terminal region and/or the amino acid substitution in FRI-Ler contribute to the functional difference between alleles (Figure 4). In fact, previous work demonstrated that a deletion of 118 residuals from the FRI $\mathrm{N}$-terminus resulted in reduced functionality. Nevertheless, plants missing that part of the protein still flowered later and revealed higher FLC expression levels than both Col-0 and transgenic lines lacking a substantial part of the FRI C-terminus [17]. Further studies used a deletion series to demonstrate that the ability of FRI to act as the scaffold protein for a $F L C$ activating complex depends mainly on its C-terminal part [18]. These studies suggest that the truncated FRI-Ler protein should be able to interact with its known partners to build the FRI-complex and, thus, to induce FLC expression. Finally, the amino acid change from leucine in FRIwt to isoleucine in FRI-Ler in the first coiled-coil domain is very conservative, but might impair the functionality of the protein (Figure 1d). However, the small differences observed between the FRI-Ler and FRI-wt alleles expressed under the same promoter suggest that if real, the effect of these mutations on flowering time is minor compared to the effect of their differences in expression.
Despite the functional difference between the two alleles, accessions of the FRI-Col and FRI-Ler group are all rapid cycling and, thus, do not display a difference in their general life history strategy. Furthermore, differences in fitness traits between both allelic groups could not be detected in the present study. A more comprehensive analysis of larger data sets collected in multiple environments might be required to detect a distinct effect of the FRI-Ler allele in nature. In fact, [46] have demonstrated that, depending on the seasonal timing of specific environmental signals, Arabidopsis accessions in nature are capable of both life history strategies. For instance, Col-0 growing during autumn in the field at a specific location in Germany (Halle) displayed a winter-annual habit and flowered almost at the same time as the introgression line $\mathrm{Col}$ FRI-Sf2 that carries a functional FRI-wt allele [46]. In contrast, Col FRI-Sf2 exhibited an unexpected rapidcycling phenotype when grown in summer at one location in England (Norwich). Nevertheless, Ler resembled Col-0 with regard to flowering time at all locations tested [46]. Furthermore, we cannot exclude that the FRI-Ler allele has a role in the modulation of adaptive parameters other than flowering time and vernalization response. For example, pleiotropic effects of FRI have been reported for traits such as water use efficiency and leaf senescence, where the latter is closely linked to plant reproduction $[47,48]$.

\section{Conclusions}

In the present study we disprove the common assumption that the widespread FRI allele found in the Arabidopsis Ler accession is not functional. We demonstrate that the FRI-Ler allele increases flowering time and FLC expression and induces vernalization responses to levels that are in between the ones observed for the true null FRI-Col allele and the fully functional FRI-wt allele.

These intermediate phenotypes observed in plants carrying FRI-Ler could be explained by the presence of a close to full-length protein never before associated with this allele. We show that the differences in functionality between the FRI-Ler and the FRI-wt alleles are largely due to expression polymorphisms, although variation in the protein sequence may also play a role.

Using the limited data available from plants grown under natural conditions, we cannot conclude that the FRI-Ler allele confers differences in fitness or life history strategies when compared to the FRI-Col allele. On the other hand, phenotypic differences between accessions carrying these alleles are clear and could be advantageous under specific natural environments not explored so far.

In summary, we demonstrate the existence of an allelic series in FRI. Besides completely functional and nonfunctional alleles, we have found a widespread allelic class 
with intermediate functionality. The use of this novel classification will increase the accuracy of adaptive studies in Arabidopsis.

\section{Methods}

\section{Analysis of published datasets}

Leaf number from Recombinant Inbred Lines (RILs) in the Ler $\mathrm{x}$ Col population [31] was analyzed by grouping lines according to their genotypes at the closest molecular markers to FRI (m506, chromosome 4 at $0.0 \mathrm{cM})$ and $F L C$ (g4560, chromosome 5 at $17.3 \mathrm{cM}$ ). Phenotypic and genotypic data for these individuals was kindly provided by Johan W. van Ooijen and Caroline Dean.

Responses to vernalization from 126 Arabidopsis accessions were obtained from published data [22] and analyzed by subtracting days to flowering with vernalization from days to flowering without vernalization (plants grown in controlled environment rooms with 16 hour light). In this dataset, FRI alleles are classified as FRI-Ler, FRI-Col, nonfunctional alleles or novel alleles. The rest of the alleles were assumed to be functional $(F R I-w t)$ as they do not contain the Ler or Col-0 indels and the accessions that carry them are late flowering. For our analysis, we considered only those accessions containing alleles classified as FRI-wt, FRI-Ler or FRI-Col. Accessions for which the $F L C$ allele was classified as non-functional or novel were removed.

Expression data for $F R I$ and $F L C$ was obtained from available repositories holding northern hybridization quantification for 137 Arabidopsis accessions (greenhouse grown, $16 \mathrm{~h}$ light [38]). Accessions in this dataset were classified according to their FRI alleles by combining genotypes from the following sources: $[14,21,22,27,37]$. The assignment of $F R I$ alleles for each accession can be found in Additional file 5.

Fitness data was obtained from Arabidopsis accessions grown in four different field locations [40]. These accessions were classified according to their $F R I$ alleles as above.

\section{Sequencing of $F R I$ in Col and Ler}

Genomic DNA of Col-0 and Ler-1 was extracted from young leaves using a Plant DNeasy Mini Kit (Qiagen, Chatsworth, CA, USA). A region of approximately $3250 \mathrm{bp}$ including the complete FRI gene (AT4G00650) and an upstream and downstream region was amplified in overlapping fragments of 600-700 bp using Phusion High-Fidelity DNA Polymerase (New England Biolabs). As a reference, the same region was sequenced for the accession Bil-7 carrying a fully functional FRI-wt allele [14]. Pooled PCR products from four independent reactions were purified using QIAquick PCR purification kit (Qiagen, Chatsworth, CA, USA) and sequenced via Sanger sequencing at the Max Planck Genome Centre Cologne. The individual sequences were assembled and aligned against the sequence of Bil-7 using SeqMan Pro (DNAstar, Madison, WI, USA).

\section{Cloning and phenotyping of transgenics}

In order to clone the FRI-Ler, FRI-wt and FRI-Col alleles, we designed primers flanking positions -1372 to +2691 relative to the FRI start codon annotated in TAIR10, which include the complete gene plus an upstream region of $1061 \mathrm{bp}$ and a downstream region of $379 \mathrm{bp}$. This region was amplified by PCR from DNA of the accessions Ler-1, Bil-7 and Col-0 using Phusion High-Fidelity DNA Polymerase (New England Biolabs). For FRI-Ler and FRI-Col, PCR fragments were introduced into the binary vector pCAMBIA2300 making use of EcoRI/BamHI restriction sites. For FRI-wt, the PCR fragment was introduced into binary vector pBinGlyRed1 (kindly provided by Ed Cahoon, University of Nebraska) making use of the same EcoRI/BamHI restriction sites.

Promoter-swap constructs were generated using the MultiSite Gateway ${ }^{\circledR}$ Pro 2.0 system (Life Technologies) and genomic DNA from the accessions Ler-1 (FRI-Ler) and Bil-7 (FRI-wt). The FRI-Ler promoter region was cloned starting from position -1372 , relative to the annotated FRI start codon in TAIR10, and ending at position +126, which is right before the first ATG downstream of the indel described in FRI-Ler. For FRI$w t$, the promoter region cloned ranged between positions -1372 and -1 . Both promoter sequences were introduced into pDONRTM $221 \mathrm{P} 1-\mathrm{P} 5 \mathrm{r}$ by BP reaction. Similarly, the coding sequences for both alleles together with $379 \mathrm{bp}$ of its downstream sequence (position +1 to position +2691 bp for FRI-wt and position +127 to position +2691 for FRI-Ler) were cloned and introduced into pDONRTM 221 P5-P2. Finally, FRI$w t:: F R I-L e r$ and FRI-Ler::FRI-wt constructs were generated from LR reactions using the binary vector pFAST.

All final constructs were transformed into $E$. coli strain Top10 (One Shot ${ }^{\circledR}$ TOP10 chemically competent E. coli, Invitrogen). Inserts from positive colonies were sequenced and verified by comparing them to the PCR template sequence or to the expected in silico constructs. Subsequently, all constructs were transformed into Agrobacterium tumefaciens strain GV3101 and used for transformation of Col-0 plants by the floral dip method [49].

Transgenic plants carrying FRI-Ler or FRI-Col constructs (in PCAMBIA) were selected on MS agar plates containing $50 \mathrm{mg} / \mathrm{l}$ kanamycin. Independent $\mathrm{T}_{1}$ plants carrying a single insertion were identified and homozygous $\mathrm{T}_{3}$ plants were used for subsequent analyses. Flowering time in $\mathrm{T}_{3}$ lines carrying FRI-Col or FRI-Ler was measured in the greenhouse under long day conditions ( $16 \mathrm{~h}$ day length) with and without vernalization treatment for four weeks at $4{ }^{\circ} \mathrm{C}$.

$\mathrm{T}_{1}$ plants carrying the promoter swap between FRI-Ler and FRI-wt (in pFAST) and those carrying the Bil-7 
FRI-wt alleles (in pBinGlyRed1) were selected by their red fluorescence under a fluorescence stereomicroscope (Leica MZ16F, Leica Microsystems, Germany) using green light of wavelength about $580 \mathrm{~nm}$. Flowering time was recorded in $\mathrm{T}_{1}$ lines carrying the $F R I-w t$ allele and the promoter swap lines FRI-wt:FRI-Ler and FRI-Ler:: $F R I-w t$ under long day condition in an environmental chamber. This experiment included plants from two independent $\mathrm{T}_{3}$ lines carrying the FRI-Ler::FRI-Ler allele (lines $14-1$ and 16-4 in Figure 2). Using these $\mathrm{T}_{3}$ lines allowed us to sow all the plants in the experiment directly on soil, as FRI-Ler $\mathrm{T}_{1}$ lines do not have red fluorescent marker and would have required selection on kanamycin plates.

In all experiments, flowering time was quantified as total leaf number (rosette + cauline) on the day the first flower opened.

\section{Expression analysis using quantitative RT-PCR}

For expression analysis of transgenics, seedlings (leaves plus shoot) grown in an environmental chamber at long day conditions (16 h light) were harvested 10 days after sowing. Material of 8 to 10 seedlings was pooled for each of three biological replicates. RNA was extracted using Trizol (Ambion ${ }^{\circledR}$ TRIzol $^{\circledR}$ RNA Isolation Reagent, Life Technologies) and transcribed into cDNA using Super Script ${ }^{\circledR}$ II Reverse Transcriptase (Invitrogen). Quantitative RT-PCR was performed on a CFX384 Touch $^{\text {tw }}$ Real-Time PCR Detection System using SYBR Green dye $\left(\mathrm{iQ}^{\mathrm{m}} \mathrm{SYBR}^{\circledR}\right.$ Green Supermix, Biorad) using the following gene-specific primers: $F L C$-fwd: 5'-CCGAACTCATGTTGAAGCTTGTTGAG3', FLC-rev: 5' - CGGAGATTTGTCCAGCAGGTG-3', FRI-fwd: 5' - TGCCTGATCGTGGTAAAGGGAAG-3' and FRI-rev: 5' - AGCACCGGCAATCTCATTCGAAC-3'. Expression values were determined using the standard curve method and normalized to the expression of $P P 2 A$ ( $P P 2 A$ fwd: 5'-TAACGTGGCCAAAATGATGC-3', $P P 2 A$-rev: 5' GTTCTCCACAACCGCTTGGT-3'). Normalized expression was averaged for three biological replicates each analyzed in two or three technical replicates.

\section{Allele-specific expression analysis}

Leaves from $\mathrm{F}_{1}$ hybrids involving the Ler-1 parent were collected at bolting from plants grown in $12 \mathrm{~h}$ days in a controlled environmental chamber. Material from $\mathrm{F}_{1}$ hybrids involving the Col-0 parent were collected from ten-day old seedlings grown in long days ( $16 \mathrm{~h}$ light) in a controlled environmental chamber. Here, material of 8 to 10 seedlings was pooled per biological replicate. RNA was extracted from $20 \mathrm{mg}$ of frozen material using RNeasy Plant kit (Qiagen) combined with on-column DNase digestion using RNase-Free DNase Set (Qiagen). Subsequently, cDNA was synthesized using SuperScript ${ }^{\circledR}$ III Reverse Transcriptase kit (Invitrogen) in the presence of RNase inhibitor
RNasin (Promega) following specifications from the manufacturer. Genomic DNA was extracted from the same samples using DNease Plant kit (Qiagen).

PCR distinguishing FRI-Ler versus FRI-wt alleles targeted a variant from $C$ (in Bor-4, Bur-0, Sha and Knox10) to A (in Ler-1) at chromosome 4, position 269257 (TAIR10). The fragment containing this SNP was amplified using primers 5'Biotin-TCAGTTGCAGTGGAAA CATTCA-3' and 5'-GCGTTTTCGATTGACTCGATG T-3', and pyrosequencing was performed using primer 5'-TGACTCGATGTGCTTCT-3'. To distinguish the FRI-Ler and FRI-Col alleles in the Ler $\mathrm{x}$ Col $\mathrm{F}_{1}$ hybrid we targeted a variant from A in Col-0 to G in Ler-1 at chromosome 4, position 269469 (TAIR10). The fragment containing this SNP was amplified using primers 5'Biotin-ATTGTACCGGAGACGTCGAATAA-3' and 5'-GGCCAATTTCAAAGCTGAAG-3', and pyrosequencing was performed with primer $5{ }^{\prime}$-CTTTGCTA CACATCAACTC-3'. $0.5 \mu \mathrm{L}$ of cDNA was used for PCR, which was conducted in a solution (2.5 units of Taq polymerase, $2.5 \mathrm{mM} \mathrm{MgCl} 2,200 \mu \mathrm{M}$ dNTPs, buffer B, $0.2 \mathrm{mM}$ of each dNTP; Bio-Budget) containing $20 \mathrm{pmol}$ of each primer, in the total volume of $25 \mu \mathrm{L}$. The PCR products were obtained with 50 cycles $\left(93^{\circ} \mathrm{C}\right.$, $\left.45 \mathrm{sec} ; 60^{\circ} \mathrm{C}, 45 \mathrm{sec} ; 72^{\circ} \mathrm{C}, 1 \mathrm{~min}\right)$.

Allele-specific mRNA abundances were measured using Pyrosequencer $A B$ (Biotage $A B$, now Qiagen) following manufacturer's instructions for sample preparation and pyrosequencing reactions. Vacuum sample preparation was performed using $15 \mu \mathrm{L}$ of PCR product mixed with $5 \mu \mathrm{L}$ of Streptavidin Sepharose beads (GE Healthcare), $40 \mu \mathrm{L}$ of PyroMark Binding Buffer (Qiagen) and $20 \mu \mathrm{L}$ of LiChroSolv water (Merck). Pyrosequencing was performed in a PyroMark Q96 Plate Low (Qiagen) in each well containing $1 \mu \mathrm{L}$ of sequencing primer $(10 \mu \mathrm{M})$ and $40 \mu \mathrm{L}$ of Pyromark Annealing Buffer (Qiagen) using PyroMark Gold Q96 Reagents (Qiagen). Allele-specific expression for each SNP was estimated using the pyrosequencing software (PSQ 96MA 2.1.1, Biotage AB) based on the peak height for each allele at the SNP. A peak correction factor of 0.86 was used for incorporation of dATP $\alpha \mathrm{S}$, as recommended by the manufacturer.

\section{Availability of supporting data}

The coding DNA sequence and translated protein sequence of the FRI-Ler allele supporting the results of this article are available through NCBI's GenBank under accession number KJ545576 (http://www.ncbi.nlm.nih.gov/genbank).

\section{Additional files}

Additional file 1: Response to vernalization quantified as the decrease in the total number of leaves at flowering for Arabidopsis accessions grouped by their FRI allele, as described in [22]. 
Additional file 2: RNA-seq coverage at the $F R I$ locus in seedlings of seven Arabidopsis accessions carrying the FRI-Ler allele.

Additional file 3: Expression of $F R I$ in transgenic lines carrying the FRI transgene.

Additional file 4: Assessment of fitness differences between accessions containing FRI-Col, FRI-Ler or FRI-wt alleles.

Additional file 5: Assignment of $F R I$ alleles in Arabidopsis accessions used in Figure 3 and Additional file 4. For both articles referenced in the table [38,46], FR/ alleles are marked with y (yes) when identified in the article and with n (no) when not present in the article.

\section{Competing interests}

The authors declare that they have no competing interests.

\section{Authors' contributions}

IS cloned the natural FRI alleles and wrote the manuscript. LZ generated the promoter-swap lines, selected and phenotyped all transgenic lines and performed the expression analyses. MR conducted allele-specific expression analyses. JMJG supervised the work, analyzed the data and wrote the manuscript. All authors read and approved the final manuscript.

\section{Acknowledgements}

We thank Caroline Dean and Johan W. Ooijen for kindly providing the phenotypic and genotypic data of the Ler x Col RIL population. Furthermore, we thank Ute Tartler and Regina Gentges for plant handling. We are grateful to Maarten Koornneef, Niels Müller and Jinyong Hu for discussions during the course of the work and for comments on the manuscript. IS acknowledges support from the German Research Foundation (DFG project number SCHM2793/1-1) and the Max Planck Society. LZ was funded by an International Max Planck Research School PhD fellowship. MR received funding from the German Research Foundation under the German-Israeli Project Cooperation program (DFG DIP project number FE552/12-1).

Received: 10 March 2014 Accepted: 4 August 2014

Published: 13 August 2014

\section{References}

1. Hoffmann MH: Biogeography of Arabidopsis thaliana (L.) Heynh. (Brassicaceae). J Biogeogr 2002, 29(1):125-134.

2. Johanson U, West J, Lister C, Michaels S, Amasino R, Dean C: Molecular analysis of FRIGIDA, a major determinant of natural variation in Arabidopsis flowering time. Science 2000, 290(5490):344-347.

3. Michaels SD, Amasino RM: FLOWERING LOCUS C encodes a novel MADS domain protein that acts as a repressor of flowering. Plant Cell 1999, 11(5):949-956.

4. Deng W, Ying H, Helliwell CA, Taylor JM, Peacock WJ, Dennis ES: FLOWERING LOCUS C (FLC) regulates development pathways throughout the life cycle of Arabidopsis. Proc Natl Acad Sci U S A 2011, 108(16):6680-6685

5. Helliwell CA, Wood CC, Robertson M, James Peacock W, Dennis ES: The Arabidopsis FLC protein interacts directly in vivo with $\mathrm{SOC} 1$ and FT chromatin and is part of a high-molecular-weight protein complex. Plant J 2006, 46(2):183-192.

6. Hepworth SR, Valverde F, Ravenscroft D, Mouradov A, Coupland G: Antagonistic regulation of flowering-time gene SOC1 by CONSTANS and FLC via separate promoter motifs. EMBO J 2002, 21(16):4327-4337.

7. Andres F, Coupland $\mathrm{G}$ : The genetic basis of flowering responses to seasonal cues. Nat Rev Genet 2012, 13(9):627-639.

8. Crevillen P, Dean C: Regulation of the floral repressor gene FLC: the complexity of transcription in a chromatin context. Curr Opin Plant Biol 2011, 14(1):38-44.

9. Bastow R, Mylne JS, Lister C, Lippman Z, Martienssen RA, Dean C: Vernalization requires epigenetic silencing of $F L C$ by histone methylation. Nature 2004, 427(6970):164-167.

10. Heo JB, Sung S: Encoding memory of winter by noncoding RNAs. Epigenetics 2011, 6(5):544-547.

11. Swiezewski S, Liu F, Magusin A, Dean C: Cold-induced silencing by long antisense transcripts of an Arabidopsis Polycomb target. Nature 2009, 462(7274):799-802.
12. Angel A, Song J, Dean C, Howard M: A Polycomb-based switch underlying quantitative epigenetic memory. Nature 2011, 476(7358):105-108.

13. Coustham V, Li P, Strange A, Lister C, Song J, Dean C: Quantitative modulation of polycomb silencing underlies natural variation in vernalization. Science 2012, 337(6094):584-587.

14. Shindo C, Aranzana MJ, Lister C, Baxter C, Nicholls C, Nordborg M, Dean C: Role of FRIGIDA and FLOWERING LOCUS $C$ in determining variation in flowering time of Arabidopsis. Plant Physiol 2005, 138(2):1163-1173.

15. Caicedo AL, Stinchcombe JR, Olsen KM, Schmitt J, Purugganan MD: Epistatic interaction between Arabidopsis FRI and FLC flowering time genes generates a latitudinal cline in a life history trait. Proc Natl Acad Sci U S A 2004, 101(44):15670-15675.

16. Sheldon CC, Conn AB, Dennis ES, Peacock WJ: Different regulatory regions are required for the vernalization-induced repression of FLOWERING LOCUS C and for the epigenetic maintenance of repression. Plant Cell 2002, 14(10):2527-2537.

17. Risk JM, Laurie RE, Macknight RC, Day CL: FRIGIDA and related proteins have a conserved central domain and family specific $\mathrm{N}$ - and C-terminal regions that are functionally important. Plant Mol Biol 2010, 73(4-5):493-505.

18. Choi K, Kim J, Hwang HJ, Kim S, Park C, Kim SY, Lee I: The FRIGIDA complex activates transcription of FLC, a strong flowering repressor in Arabidopsis, by recruiting chromatin modification factors. Plant Cell 2011, 23(1):289-303.

19. Jiang $D, G u X, H e Y$ : Establishment of the winter-annual growth habit via FRIGIDA-mediated histone methylation at FLOWERING LOCUS C in Arabidopsis. Plant Cell 2009, 21(6):1733-1746.

20. Geraldo N, Baurle I, Kidou S, Hu X, Dean C: FRIGIDA delays flowering in Arabidopsis via a cotranscriptional mechanism involving direct interaction with the nuclear cap-binding complex. Plant Physiol 2009, 150(3):1611-1618.

21. Hagenblad J, Tang C, Molitor J, Werner J, Zhao K, Zheng H, Marjoram P, Weigel D, Nordborg M: Haplotype structure and phenotypic associations in the chromosomal regions surrounding two Arabidopsis thaliana flowering time loci. Genetics 2004, 168(3):1627-1638.

22. Lempe J, Balasubramanian S, Sureshkumar S, Singh A, Schmid M, Weigel D: Diversity of flowering responses in wild Arabidopsis thaliana strains. PLoS Genet 2005, 1(1):109-118.

23. Le Corre V, Roux F, Reboud X: DNA polymorphism at the FRIGIDA gene in Arabidopsis thaliana: extensive nonsynonymous variation is consistent with local selection for flowering time. Mol Biol Evol 2002, 19(8):1261-1271

24. Mendez-Vigo B, Pico FX, Ramiro M, Martinez-Zapater JM, Alonso-Blanco C: Altitudinal and climatic adaptation is mediated by flowering traits and FRI, FLC, and PHYC genes in Arabidopsis. Plant Physiol 2011, 157(4):1942-1955.

25. Gazzani S, Gendall AR, Lister C, Dean C: Analysis of the molecular basis of flowering time variation in Arabidopsis accessions. Plant Physiol 2003, 132(2):1107-1114.

26. Stinchcombe JR, Weinig C, Ungerer M, Olsen KM, Mays C, Halldorsdottir SS, Purugganan MD, Schmitt J: A latitudinal cline in flowering time in Arabidopsis thaliana modulated by the flowering time gene FRIGIDA. Proc Natl Acad Sci U S A 2004, 101(13):4712-4717.

27. Toomajian C, Hu TT, Aranzana MJ, Lister C, Tang C, Zheng H, Zhao K Calabrese P, Dean C, Nordborg M: A nonparametric test reveals selection for rapid flowering in the Arabidopsis genome. PLoS Biol 2006, 4(5):e137.

28. Werner JD, Borevitz JO, Uhlenhaut NH, Ecker JR, Chory J, Weigel D: FRIGIDA-independent variation in flowering time of natural Arabidopsis thaliana accessions. Genetics 2005, 170(3):1197-1207.

29. El-Lithy ME, Bentsink L, Hanhart CJ, Ruys GJ, Rovito D, Broekhof JL, van der Poel HJ, van Eijk MJ, Vreugdenhil D, Koornneef M: New Arabidopsis recombinant inbred line populations genotyped using SNPWave and their use for mapping flowering-time quantitative trait loci. Genetics 2006, 172(3):1867-1876.

30. Tisne S, Schmalenbach I, Reymond M, Dauzat M, Pervent M, Vile D, Granier $C$ : Keep on growing under drought: genetic and developmental bases of the response of rosette area using a recombinant inbred line population. Plant Cell Environ 2010, 33(11):1875-1887.

31. Jansen RC, Van Ooijen JW, Stam P, Lister C, Dean C: Genotype-by-environment interaction in genetic mapping of multiple quantitative trait loci. Theor App/ Genet 1995, 91(1):33-37. 
32. Koornneef M, Blankestijn-de Vries H, Hanhart C, Soppe W, Peeters T: The phenotype of some late-flowering mutants is enhanced by a locus on chromosome 5 that is not effective in the Landsberg erecta wild-type. Plant J 1994, 6(6):911-919.

33. Liu J, He Y, Amasino $R$, Chen $X$ : siRNAs targeting an intronic transposon in the regulation of natural flowering behavior in Arabidopsis. Genes Dev 2004, 18(23):2873-2878.

34. Lee I, Amasino RM: Effect of vernalization, photoperiod, and light quality on the flowering phenotype of arabidopsis plants containing the FRIGIDA gene. Plant Physiol 1995, 108(1):157-162.

35. Lee I, Bleecker A, Amasino R: Analysis of naturally occurring late flowering in Arabidopsis thaliana. Mol Gen Genet 1993, 237(1-2):171-176.

36. Gan X, Stegle O, Behr J, Steffen JG, Drewe P, Hildebrand KL, Lyngsoe R, Schultheiss SJ, Osborne EJ, Sreedharan VT, Kahles A, Bohnert R, Jean G, Derwent P, Kersey P, Belfield EJ, Harberd NP, Kemen E, Toomajian C, Kover PX, Clark RM, Rätsch G, Mott R: Multiple reference genomes and transcriptomes for Arabidopsis thaliana. Nature 2011, 477(7365):419-423.

37. Korves TM, Schmid KJ, Caicedo AL, Mays C, Stinchcombe JR, Purugganan $M D$, Schmitt J: Fitness effects associated with the major flowering time gene FRIGIDA in Arabidopsis thaliana in the field. Am Nat 2007, 169(5):E141-E157.

38. Atwell S, Huang YS, Vilhjalmsson BJ, Willems G, Horton M, Li Y, Meng D, Platt A, Tarone AM, Hu TT, Jiang R, Muliyati NW, Zhang X, Amer MA, Baxter I, Brachi B, Chory J, Dean C, Debieu M, de Meaux J, Ecker JR, Faure N, Kniskern JM, Jones JDG, Michael T, Nemri A, Roux F, Salt DE, Tang C, Todesco M, et al: Genome-wide association study of 107 phenotypes in Arabidopsis thaliana inbred lines. Nature 2010, 465(7298):627-631.

39. Wittkopp PJ, Haerum BK, Clark AG: Evolutionary changes in cis and trans gene regulation. Nature 2004, 430(6995):85-88.

40. Fournier-Level A, Korte A, Cooper MD, Nordborg M, Schmitt J, Wilczek AM: A map of local adaptation in Arabidopsis thaliana. Science 2011, 334(6052):86-89.

41. Ehrenreich IM, Hanzawa Y, Chou L, Roe JL, Kover PX, Purugganan MD: Candidate gene association mapping of Arabidopsis flowering time. Genetics 2009, 183(1):325-335.

42. Lee I, Aukerman MJ, Gore SL, Lohman KN, Michaels SD, Weaver LM, John MC, Feldmann KA, Amasino RM: Isolation of LUMINIDEPENDENS: a gene involved in the control of flowering time in Arabidopsis. Plant Cell 1994, 6(1):75-83.

43. Macknight R, Bancroft I, Page T, Lister C, Schmidt R, Love K, Westphal L, Murphy G, Sherson S, Cobbett C, Dean C: FCA, a gene controlling flowering time in Arabidopsis, encodes a protein containing RNA-binding domains. Cell 1997, 89(5):737-745.

44. Simpson GG, Dijkwel PP, Quesada V, Henderson I, Dean C: FY is an RNA 3' end-processing factor that interacts with FCA to control the Arabidopsis floral transition. Cell 2003, 113(6):777-787.

45. Schlappi MR: FRIGIDA LIKE 2 is a functional allele in Landsberg erecta and compensates for a nonsense allele of FRIGIDA LIKE 1. Plant Physiol 2006, 142(4):1728-1738.

46. Wilczek AM, Roe JL, Knapp MC, Cooper MD, Lopez-Gallego C, Martin LJ, Muir CD, Sim S, Walker A, Anderson J, Egan JF, Moyers BT, Petipas R, Giakountis A, Charbit E, Coupland G, Welch SM, Schmitt J: Effects of genetic perturbation on seasonal life history plasticity. Science 2009, 323(5916):930-934.

47. Wingler A, Purdy SJ, Edwards SA, Chardon F, Masclaux-Daubresse C: QTL analysis for sugar-regulated leaf senescence supports floweringdependent and -independent senescence pathways. New Phytol 2010, 185(2):420-433.

48. Lovell JT, Juenger TE, Michaels SD, Lasky JR, Platt A, Richards JH, Yu X, Easlon HM, Sen S, McKay JK: Pleiotropy of FRIGIDA enhances the potential for multivariate adaptation. Proc Biol Sci/ Royal Soc 2013, 280 (1763):20131043.

49. Clough SJ, Bent AF: Floral dip: a simplified method for Agrobacteriummediated transformation of Arabidopsis thaliana. Plant J 1998, 16(6):735-743.

\section{Submit your next manuscript to BioMed Central and take full advantage of:}

- Convenient online submission

- Thorough peer review

- No space constraints or color figure charges

- Immediate publication on acceptance

- Inclusion in PubMed, CAS, Scopus and Google Scholar

- Research which is freely available for redistribution 